\title{
A New Technique for Accurate and Stable Modeling of Arbitrarily Oriented Thin Wires in the FDTD Method
}

\author{
Fredrik Edelvik \\ Department of Information Technology, Scientific Computing, \\ Uppsala University, Box 337, SE-751 05 Uppsala, Sweden, \\ fredrik@tdb.uu.se
}

\begin{abstract}
A subcell model for thin wires in the finite-difference time-domain (FDTD) method using modified telegraphers equations has been developed by Holland et al. In this paper we present an extension of their algorithm, which allows arbitrarily located and oriented wires with respect to the Cartesian grid. This is important to be able to accurately model wires that cannot be aligned to the Cartesian grid, e.g. tilted wires and circular loop wires. A symmetric coupling between field and wires yields a stable time-continuous fieldwire system and the fully discrete field-wire system is stable under a CFL condition. The accuracy and excellent consistency of the proposed method are demonstrated for dipole and loop antennas with comparisons with the Method of Moments and experimental data.
\end{abstract}

\section{Introduction}

The ability to model features that are small relative to the cell size is often important in electromagnetic simulations. Thus, the developement of accurate models that characterize the physics of the feature without the need for a highly resolved grid is essential.

Thin wires are often important parts of electromagnetic compatibility and antenna problems. There are basically two approaches for constructing thin wire subcell models for the finite-difference time-domain (FDTD) method. Holland et al. [1] separate the wire from the 3D volume and the current and voltage on the wire are described by modified telegraphers equations. Umashankar et al. [2] include the effect of the wire directly into the stencil of the Yee scheme.

The algorithm proposed by Holland et al. is able to treat wires that are running parallel to the edges in the Cartesian grid, but for arbitrarily oriented wires both approaches have to rely on a staircased discretization, which often introduces large errors. A generalization of the algorithm of Holland et al. to arbitrarily oriented wires has therefore been proposed by Ledfelt [3]. However, for bent wires this generalization suffers from problems with undamped low frequency oscillations.

Riley has developed a method that incorporates thin wires into the finite-element timedomain (FETD) method by discretizing a second-order wave equation for the current [4]. The method requires the wires to be aligned with the edges in the unstructured grid and may suffer from late time instability problems. In [5] an unconditionally stable method for incorporating arbitrarily oriented wires into the FETD method is developed. In this paper we will present a method similar to the one in [5] for arbitrarily oriented wires in FDTD. The outline of the rest of the paper is as follows: In the next section we introduce the equations and their discretization using the leap-frog scheme. In the following section we take a closer look at the coupling between field and wire and show how the algorithm proposed by Holland et al. can be generalized to take arbitrarily oriented wires into account. In Section 4 we prove that the time-continuous field-wire system is stable. The following section discusses the possibility of treating wires in a hybrid FDTD-FETD 
solver. In the results section the proposed method is applied to different configurations of dipole and loop antennas. The final section concludes the paper and discusses future extensions.

\section{Governing equations}

The Maxwell equations for linear, isotropic and non-dispersive media are given by

$$
\begin{aligned}
& \mu \frac{\partial \boldsymbol{H}}{\partial t}+\nabla \times \boldsymbol{E}=0, \\
& \epsilon \frac{\partial \boldsymbol{E}}{\partial t}-\nabla \times \boldsymbol{H}=-\sigma \boldsymbol{E}-\boldsymbol{J},
\end{aligned}
$$

where $\boldsymbol{E}$ is the electric field, $\boldsymbol{H}$ is the magnetic field, $\boldsymbol{J}$ is the electric current density, $\epsilon$ is the electric permittivity, $\mu$ is the magnetic permeability and $\sigma$ is the electric conductivity.

To derive the wire equation we study an infinitely long cylinder of radius $a$ running in the $\xi$-direction. Following Holland et al. [1] we obtain in cylindrical coordinates, see Figure 1,

$$
\begin{aligned}
C \frac{\partial V}{\partial t} & =-C \frac{\sigma}{\epsilon} V-\frac{\partial I}{\partial \xi}, \\
L \frac{\partial I}{\partial t} & =E_{\xi}+\tilde{V}^{i n c}-R I-\frac{\partial V}{\partial \xi},
\end{aligned}
$$

where $I$ is the wire current, $V$ is the wire voltage, $E_{\xi}$ is the electric field along the wire, $\tilde{V}^{i n c}$ is a voltage source per unit length, $R$ is the wire resistance per unit length, $L$ is the wire inductance per unit length and $C$ is the wire conductance per unit length.

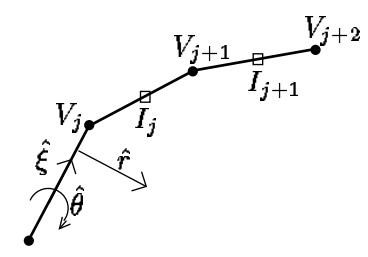

Figure 1: Three segments of a discretized wire described in cylindrical coordinates.

The current vanishes at an open termination, whereas the spatial derivative of the current vanishes when the wire terminates on a large perfect electric conductor. The first-order Mur nonreflecting boundary condition is used to simulate a half-infinite wire.

The field equations (1-2) are discretized by the well known FDTD method [6]. The wire equations (3-4) are discretized by a leap-frog scheme on a staggered grid. The unknown voltages are located at the nodes of the wire, whereas the unknown currents are located at the midpoint of the wire segments, see Figure 1. If we assume that $R=0$ and $\sigma=0$, the discretized wire equations are given by

$$
\begin{aligned}
& V_{j}^{n+1}=V_{j}^{n}-\frac{\Delta t}{C} \frac{I_{j}^{n+\frac{1}{2}}-I_{j-1}^{n+\frac{1}{2}}}{\Delta \xi}, \\
& I_{j}^{n+\frac{1}{2}}=I_{j}^{n-\frac{1}{2}}-\frac{\Delta t}{L}\left[\frac{V_{j+1}^{n}-V_{j}^{n}}{\Delta \xi}-E_{\xi j}^{\text {interp }}+\frac{V_{j}^{i n c}}{\Delta \xi}\right],
\end{aligned}
$$

where $\Delta \xi$ is the length of the wire segments which are assumed equal to simplify the description and $V^{i n c}$ is a voltage beam source. The inductance per unit length is approximated as

$$
L=\frac{\mu}{2 \pi} \log \frac{r_{0}+a}{2 a},
$$


where $\left(r_{0}+a\right) / 2$ is an average distance from the wire to the surrounding electric fields used to drive the wire (cf. Section 3 ). Note that $L$ is positive as long as $r_{0}>a$, which is necessary in order to have a well posed problem.

\section{Coupling between the wire and the surrounding field}

The coupling between the field equations (1-2) and the wire equations (3-4) are through the source terms $\boldsymbol{J}$ and $E_{\xi}$. Since we are focussing on wires which are not aligned with the edges in the grid we need to approximate the electric field along the wire through interpolation of the surrounding field components. The current density, on the other hand, is approximated by distributing the current from the wire to the surrounding field components.

The current $I$ along the wire can be expanded in basis functions as

$$
I(\xi)=\sum_{j} I_{j} \Phi_{j}(\xi)
$$

where $\Phi_{j}$ is a constant basis function equal to one on segment $j$ and zero on all other segments, and $I_{j}$ is the unknown current at wire segment $j$. The current density $\boldsymbol{J}$ is now expressed as

$$
\boldsymbol{J}(r, \xi)=I(\xi) g(r) \hat{\xi}=\sum_{j} I_{j} \Phi_{j}(\xi) g(r) \hat{\xi},
$$

where $r$ is a radial distance from the wire and $g(r)$ is a weighting function satisfying

$$
\int_{r \geq a} g(r) 2 \pi r d r=1
$$

and thus has dimension $\left[\frac{1}{m^{2}}\right]$. Furthermore, it is important that this function decreases with $r$ and equals zero for $r \geq r_{0}$, which gives a compact support. The function used in this paper is defined as

$$
g(r)= \begin{cases}0, & r<a, \\ \frac{1+\cos \left(\frac{\pi r}{r_{0}}\right)}{\pi\left(r_{0}^{2}-a^{2}\right)+\frac{2 r_{0}^{2}}{\pi}\left(-1-\cos \frac{\pi a}{r_{0}}-\frac{\pi a}{r_{0}} \sin \frac{\pi a}{r_{0}}\right)}, & a \leq r \leq r_{0}, \\ 0, & r>r_{0},\end{cases}
$$

where $r_{0}=1.7 \times \max (\Delta x, \Delta y, \Delta z)$ is thus a grid dependent radial distance from the wire.

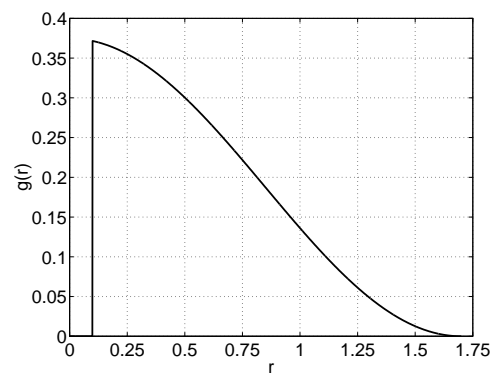

Figure 2: The weighting function $g(r)$ for $r_{0}=1.7$ and $a=0.1$ (right).

The electric field is expanded in basis functions as

$$
\boldsymbol{E}(\boldsymbol{r})=\sum_{k} E_{k} \boldsymbol{N}_{k}(\boldsymbol{r})
$$


where $\boldsymbol{N}_{k}$ are edge basis functions for hexahedral elements, see [7]. The sum is taken over all edges in the grid and $E_{k}$ are the electric fields along the edges. Note that the FEM terminology is used in order to get a more compact notation. The approximation of the electric field inside a hexahedra according to (12) is nothing but a trilinear interpolation of the involved edge values. If we multiply $E_{\xi}$ with $g(r) \Phi_{j}(\xi)$ we can approximate the electric field along wire segment $j$ as

$$
E_{\xi j}^{\text {interp }}=\frac{\int_{V} E_{\xi}(\boldsymbol{r}) g(r) \Phi_{j}(\xi) d V}{\Delta \xi}=\frac{\sum_{k} E_{k} \int_{V} \hat{\xi} \cdot \boldsymbol{N}_{k}(\boldsymbol{r}) g(r) \Phi_{j}(\xi) d V}{\Delta \xi}=\frac{(\mathcal{P} E)_{j}}{\Delta \xi} .
$$

The volume integral is taken over all space, but due to the compact support of $\boldsymbol{N}_{k}(\boldsymbol{r})$, $g(r)$ and $\Phi_{j}(\xi)$, only electric field components in a neighborhood of segment $j$ contributes. The integral in (13) is calculated using a Gaussian quadrature formula.

In the same manner $J_{k}^{\text {distrib }}$ is approximated by

$$
J_{k}^{d i s t r i b}=\frac{\int_{V} \boldsymbol{J}(\boldsymbol{r}) \cdot \boldsymbol{N}_{k}(\boldsymbol{r}) d V}{\Delta x \Delta y \Delta z}=\frac{\sum_{j} I_{j} \int_{V} \hat{\xi} \cdot \boldsymbol{N}_{k}(\boldsymbol{r}) \Phi_{j}(\xi) g(r) d V}{\Delta x \Delta y \Delta z}=\frac{\left(\mathcal{P}^{T} I\right)_{k}}{\Delta x \Delta y \Delta z},
$$

where the division by $\Delta x \Delta y \Delta z$ is explained by the fact that this is the volume of the dual cell surrounding $J_{k}^{\text {distrib }}$. Note that apart from a scale factor we have a skew-symmetric coupling between field and wire.

For a straight wire each wire segment has a corresponding interpolation cylinder of radius $r_{0}$, which determines the domain of support. For bent wires it has been found crucial to slightly modify the domain of support in order to avoid undamped low frequency oscillations. We want to avoid that cylinders corresponding to neighboring segments overlap and that we have regions outside bends which are not covered by any cylinder. An overlap implies that too much current is distributed from the wire to this region, and a gap means that no current is distributed to this region. In Figure 3 we show the projection of the cylinder on the plane with normal $\hat{n}=\hat{\xi}_{b 2} \times \hat{\xi}_{b 1}$. The modified cylinder corresponding to segment $j-1$ is bounded by planes with normals $\hat{\xi}_{b 1}$ and $\hat{\xi}_{b 1}+\hat{\xi}_{b 2}$, respectively. The overlap below and the gap above the $V_{j}$ node are indicated by dashed lines in Figure 3 .

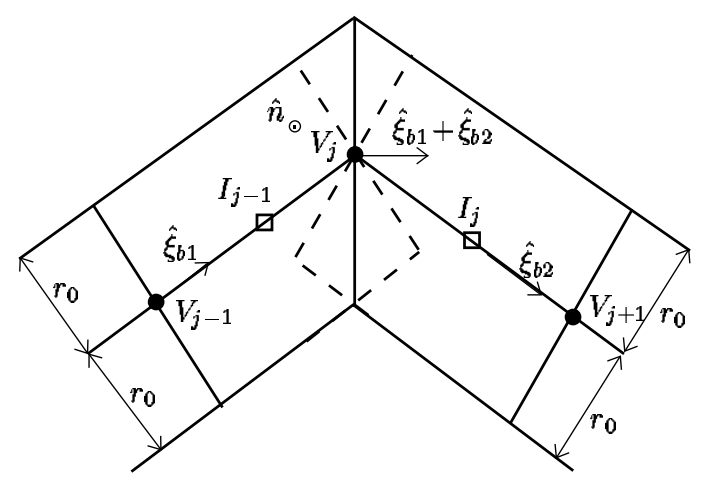

Figure 3: A slice of the interpolation cylinders at a wire bend. The dashed lines indicate the boundaries of the unmodified cylinders.

These modifications for bent wires are necessary in order to obtain accurate results on the loop antenna in the results section and are also believed to be the major reason why the approach described in [3] does not work well for bent wires.

Some modifications of our proposed method are needed if other geometry such as e.g. a PEC object falls within the chosen radius (but here the basic thin wire assumptions become questionable). A general treatment of these issues requires further investigation. 


\section{Stability}

In this section we prove that the time-continuous field-wire system is stable. Let $\boldsymbol{H}$ contain all the magnetic field unknowns, and accordingly for $\boldsymbol{E}, \boldsymbol{I}$, and $\boldsymbol{V}$. Assume that $R=0$, $\sigma=0$, and $\Delta=\Delta_{x}=\Delta_{y}=\Delta_{z}$. We introduce the following scaling of the variables:

$$
\boldsymbol{h}=\sqrt{\mu} \boldsymbol{H}, \boldsymbol{e}=\sqrt{\epsilon} \boldsymbol{E}, \boldsymbol{i}=\frac{\sqrt{\mu} \sqrt{\tilde{L}} \sqrt{\Delta \xi}}{\Delta^{3 / 2}} \boldsymbol{I}, \boldsymbol{v}=\frac{\sqrt{\epsilon} \sqrt{\Delta \xi}}{\sqrt{\tilde{L}} \Delta^{3 / 2}} \boldsymbol{V},
$$

where we have split $L$ as $L=\mu \tilde{L}$. If we rename our variables and scaled operators, and choose the old names we can write the field-wire system on matrix form as

$$
\left(\begin{array}{c}
\dot{\boldsymbol{H}} \\
\dot{\boldsymbol{E}} \\
\dot{\boldsymbol{I}} \\
\dot{\boldsymbol{V}}
\end{array}\right)=\left(\begin{array}{cccc}
0 & \mathcal{A} & 0 & 0 \\
-\mathcal{A}^{\mathcal{T}} & 0 & -\mathcal{P}^{\mathcal{T}} & 0 \\
0 & \mathcal{P} & 0 & -\mathcal{B}^{\mathcal{T}} \\
0 & 0 & \mathcal{B} & 0
\end{array}\right)\left(\begin{array}{c}
\boldsymbol{H} \\
\boldsymbol{E} \\
\boldsymbol{I} \\
\boldsymbol{V}
\end{array}\right)
$$

where the operators takes care of the spatial discretization and interpolation and $\dot{\boldsymbol{E}}$ denotes the time derivative of $\boldsymbol{E}$. Source terms do not effect stability [8] and are therefore not included in the analysis. Let the total electromagnetic energy of the field-wire system be defined by

$$
\mathcal{E}(t)=\frac{1}{2}\left(\boldsymbol{H}^{T} \boldsymbol{H}+\boldsymbol{E}^{T} \boldsymbol{E}+\boldsymbol{I}^{T} \boldsymbol{I}+\boldsymbol{V}^{T} \boldsymbol{V}\right) .
$$

Then we have

Proposition 4.1 The time-continuous field-wire system (16) is stable in the following sense: The energy $\mathcal{E}(t)$ in (17) is preserved.

Proof Multiplying from the left by $(\boldsymbol{H} \boldsymbol{E} \boldsymbol{I} \boldsymbol{V})$ we obtain

$$
\left(\begin{array}{c}
\boldsymbol{H} \\
\boldsymbol{E} \\
\boldsymbol{I} \\
\boldsymbol{V}
\end{array}\right)^{T}\left(\begin{array}{c}
\dot{\boldsymbol{H}} \\
\dot{\boldsymbol{E}} \\
\dot{\boldsymbol{I}} \\
\dot{\boldsymbol{V}}
\end{array}\right)=\left(\begin{array}{c}
\boldsymbol{H} \\
\boldsymbol{E} \\
\boldsymbol{I} \\
\boldsymbol{V}
\end{array}\right)^{T}\left(\begin{array}{cccc}
0 & \mathcal{A} & 0 & 0 \\
-\mathcal{A}^{\mathcal{T}} & 0 & -\mathcal{P}^{\mathcal{T}} & 0 \\
0 & \mathcal{P} & 0 & -\mathcal{B}^{\mathcal{T}} \\
0 & 0 & \mathcal{B} & 0
\end{array}\right)\left(\begin{array}{c}
\boldsymbol{H} \\
\boldsymbol{E} \\
\boldsymbol{I} \\
\boldsymbol{V}
\end{array}\right)=0
$$

where the last equality follows from the fact that the matrix is skew-symmetric. Hence, we have

$$
\frac{\partial \mathcal{E}}{\partial t}=0
$$

Thus, the total electromagnetic energy is preserved and the time-continuous problem is stable.

For a conductive medium and/or a resistive wire we would have a loss of electromagnetic energy. Note that the key property for the stability of the field-wire system is that we have a skew-symmetric coupling between field and wire.

To obtain stability for the fully discrete system we also have to fulfill the CFL condition. In general it is difficult to derive the CFL condition explicitly when wires are present. However, in practise for moderately thick wires we have not seen any need to reduce the maximum time-step given by the CFL condition for FDTD without wires. A slight reduction of the time step is necessary for wires, where $a$ is significantly larger than $\Delta$, but it is doubtful whether such wires satisfy the underlying thin wire assumptions. 


\section{Treatment of thin wires in a hybrid FDTD-FETD solver}

The major drawback with the FDTD method is its inability to accurately model curved objects. This is due to the Cartesian grid, which leads to a staircase approximation of the geometry and small details are not resolved at all. An approach to avoid staircasing that has gained popularity lately is to use unstructured grids near curved objects, but revert to Cartesian grids as quickly as possible for the rest of the computational domain. We have developed a stable hybrid solver, which combines FDTD with an FETD solver [9].

We should therefore be able to handle wires which run both in the FDTD and FETD domains. In [5] an unconditionally stable method for thin wires in FETD is developed. However, this method is based on a second-order wire equation for the current, where the unknown currents are located at the nodes of the wire and an implicit timestepping scheme is used. The different positions of the unknowns in space and time make it hard to treat a wire running through the grid interface. A better approach is to discretize the first-order system (3-4) in both domains by (5) and (6). For the part of the wire inside the FDTD domain the coupling to the surrounding field is through (13) and (14). The only modification in the FETD domain is that the scaling of (14) with the volume of the dual cell is removed. This is due to the fact that FETD is based on a volume integral formulation. For wire segments close to a grid interface special care must be taken since the interpolation cylinder may include hexahedral, tetrahedral as well pyramidal elements, where the pyramidal elements are used to connect the tetrahedral and hexahedral elements [9]. Thus, to model wires running through or close to the grid interface is only a bookkeeping problem but poses no fundamental difficulties.

\section{$6 \quad$ Numerical results}

\subsection{Transmitting dipole antenna}

The first test case is a thin wire of length $41 \mathrm{~m}$ embedded in a Cartesian grid with edge lengths $\Delta=1 \mathrm{~m}$. The wire radius is $10 \mathrm{~mm}$ and it runs in the $x$-direction discretized with 41 beams. To verify the consistency of the proposed wire method we run this case for a number of different locations and orientations of the wire with respect to the volume grid. First the wire is moved transversally as in Figure 4(a) using $0.25 \Delta y\left(a_{25}\right), 0.5 \Delta y\left(a_{50}\right)$ and $0.5 \Delta y+0.5 \Delta z\left(a_{55}\right)$. Secondly, the wire is moved longitudinally as in Figure 4(b) using $0.25 \Delta x\left(b_{25}\right)$ and $0.5 \Delta x\left(b_{50}\right)$. In the third set of cases the wire is rotated in the xy-plane, Figure $4(\mathrm{c})$, by 26.6 degrees $\left(c_{27}\right)$ and 45 degrees $\left(c_{45}\right)$. Finally, the wire is rotated as in Figure 4(d) from the direction [ $\left[\begin{array}{lll}1 & 0 & 0\end{array}\right]$ to $\left[\begin{array}{lll}1 & 0.5 & 0.5\end{array}\right]\left(d_{24}\right)$ and to $\left[\begin{array}{lll}1 & 1 & 1\end{array}\right]\left(d_{35}\right)$.

The dipole antenna is excited at the midpoint using a voltage source with the shape of the differentiated Gaussian pulse

$$
f(t)=-\frac{2\left(t-t_{0}\right)}{t_{w}} e^{-\left(t-t_{0}\right)^{2} / t_{w}^{2}}, t_{0}=12.0 \times 10^{-8}, t_{w}=4.0 \times 10^{-8} .
$$

We use the time step $\Delta t=0.5 \Delta / c_{0}$. If we register the current at the midpoint we can calculate the input impedance and input admittance for the dipole antenna according to

$$
\hat{Z}_{21}(f)=\frac{\hat{V}_{21}^{i n c}(f)}{\hat{I}_{21}(f)}
$$

and

$$
\hat{Y}_{21}(f)=\frac{1}{\hat{Z}_{21}(f)}
$$




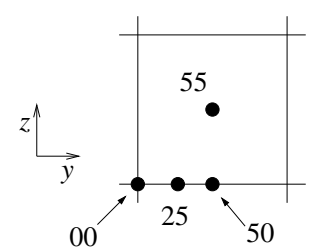

(a) Transversal translation.

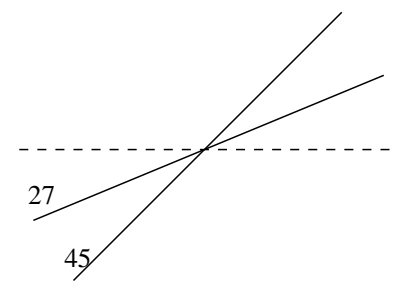

(c) Plane rotation.

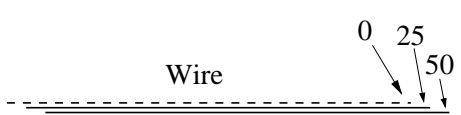

(b) Longitudinal translation.

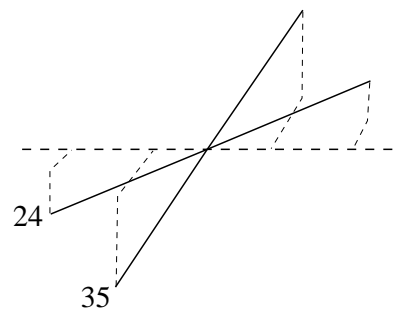

(d) 3D rotation.

Figure 4: Different locations and orientations of the dipole wire. Case a) is seen from the side, and in case b) the wire is moved in the length direction.

The real and imaginary parts of the impedance are resistance and reactance, respectively. For the admittance they are conductance and susceptance, respectively.
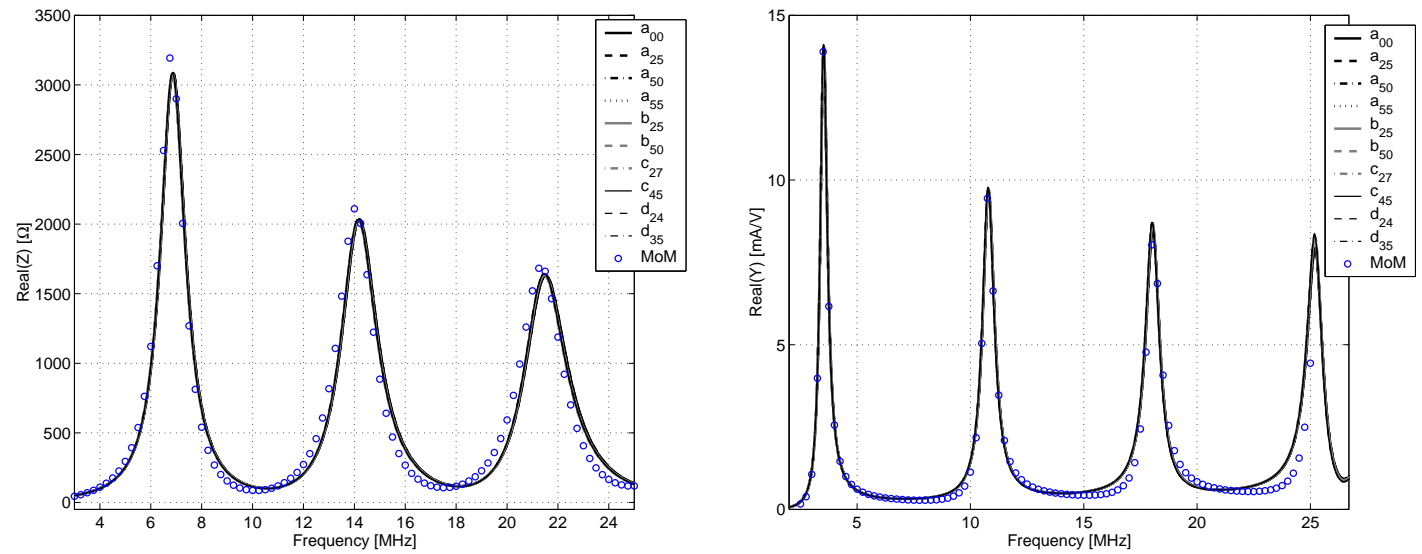

Figure 5: The input resistance for the dipole (left) and input conductance (right). A Method of Moments solution is used for comparison.

The impedance and admittance are shown in Figure 5. The results are compared to a Method of Moments solution obtained by NEC-3 and we see a good agreement for most of the frequencies. The impedance for the first half wavelength resonance should equal $73+j 42.5 \Omega$ [10]. The input impedance for the different wire setups are given in Table 1 .

We obtain very consistent results, they are a few percent less than the theoretical values, but we note that a solution obtained by NEC-3 gives $3.53 \mathrm{MHz}$ and $72.2 \Omega$, which is also slightly less than theoretical values.

\subsection{Receiving loop antenna}

In this section the thin wire model is used to simulate a circular loop antenna in receiving mode. The loop diameter is $1 \mathrm{~m}$ and the wire radius is $1.05 \mathrm{~mm}$. It is discretized using 50 


\begin{tabular}{||c||c|c||}
\hline Case & $f[\mathrm{MHz}]$ & $\operatorname{real}(\mathrm{Z})[\Omega]$ \\
\hline$a_{00}$ & 3.54 & 71.5 \\
$a_{25}$ & 3.54 & 71.4 \\
$a_{50}$ & 3.54 & 71.5 \\
$a_{55}$ & 3.54 & 71.5 \\
\hline$b_{25}$ & 3.54 & 71.5 \\
$b_{50}$ & 3.54 & 71.5 \\
\hline$c_{27}$ & 3.54 & 71.4 \\
$c_{45}$ & 3.53 & 71.2 \\
\hline$d_{24}$ & 3.53 & 71.3 \\
$d_{35}$ & 3.53 & 71.2 \\
\hline Theory & 3.66 & 73.0 \\
\hline
\end{tabular}

Table 1: The frequency and resistance at half wavelength resonance for a $41 \mathrm{~m}$ long transmitting dipole. A solution obtained by NEC-3 gives $3.53 \mathrm{MHz}$ and $72.2 \Omega$

wire segments and located in a Cartesian grid with edge lengths $\Delta=6.25 \mathrm{~cm}$. The wire loop is moved in four different ways from a basic setup (org), where the loop is centered in an xy-plane in the Cartesian grid. First the loop is moved laterally $0.25 \Delta z(a)$, secondly it is moved transversally $0.25 \Delta x$ (b) and $0.25 \Delta x+0.25 \Delta y$ (c). Thirdly it is rotated around the $\mathrm{y}$-axis by 30 degrees $\left(d_{30}\right)$ and 45 degrees $\left(d_{45}\right)$. Finally, it is rotated around the $x=y$-direction by 30 degrees $\left(e_{30}\right)$ and 45 degrees $\left(e_{45}\right)$.

The loop antenna is illuminated by a horizontally polarized plane wave. The incoming plane wave is a differentiated Gaussian pulse with $t_{w}=1.25 \times 10^{-9}$, i.e. the $-3 \mathrm{~dB}$ bandwidth is $60-350 \mathrm{MHz}$. It is designed such that the time when the wave reaches segment 26 is the same, and hence a direct comparison of the currents in this segment is possible. The time step equals $\Delta t=0.5 \Delta / c_{0}$. The current responses are shown in Figure 6.
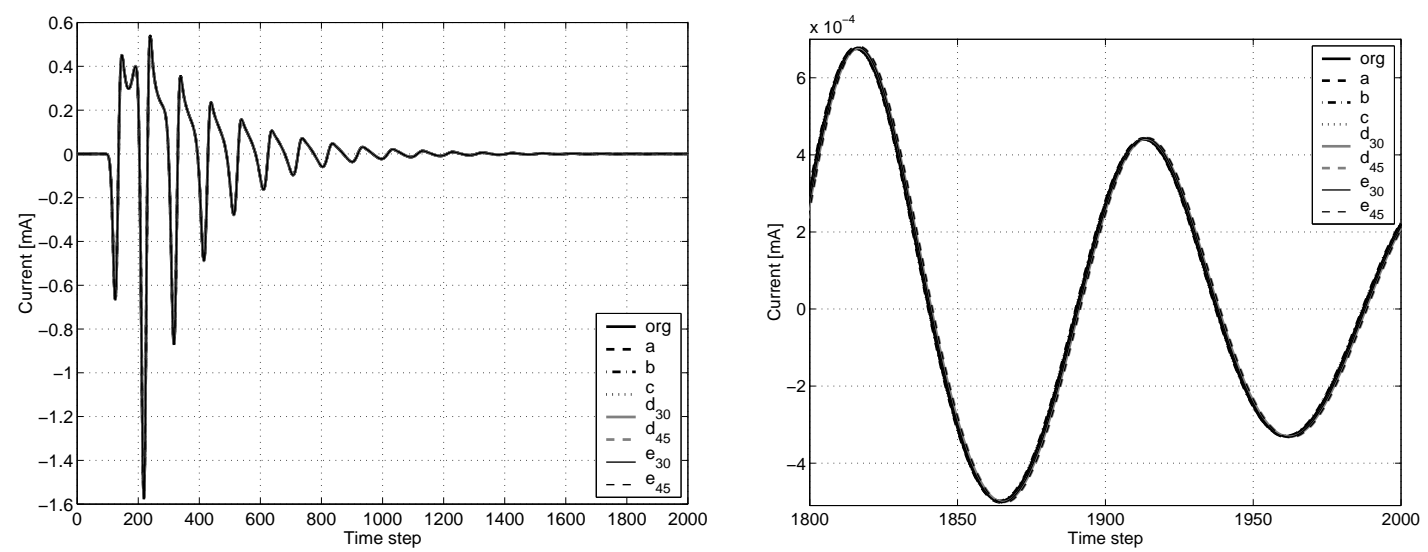

Figure 6: The current for the different wire setups.

The probed current is expanded in a Prony's series [6] and the modes corresponding to $n \lambda, n=1, \ldots, 4$, are extracted. The results are compared to a Method of Moments solution and an FETD solution [5] in Table 2. The differences between the resonance frequencies are less than $0.1 \%$ for all setups. The agreement with the FETD and MoM solutions are within $1 \%$. 


\begin{tabular}{||c||c|c|c|c||}
\hline & $f_{1}[\mathrm{MHz}]$ & $f_{2}[\mathrm{MHz}]$ & $f_{3}[\mathrm{MHz}]$ & $f_{4}[\mathrm{MHz}]$ \\
\hline org & 98.8 & 195.1 & 290.6 & 385.1 \\
$a$ & 98.8 & 195.1 & 290.6 & 385.1 \\
$b$ & 98.9 & 195.1 & 290.6 & 385.2 \\
$c$ & 98.9 & 195.1 & 290.6 & 385.1 \\
$d_{30}$ & 98.8 & 195.1 & 290.5 & 385.1 \\
$d_{45}$ & 98.8 & 195.0 & 290.5 & 385.1 \\
$e_{30}$ & 98.8 & 195.0 & 290.5 & 385.0 \\
$e_{45}$ & 98.8 & 195.0 & 290.4 & 385.0 \\
\hline FETD & 99.2 & 196.3 & 292.5 & 390.1 \\
MoM & 98.3 & 195.2 & 292.4 & 390.0 \\
\hline
\end{tabular}

Table 2: The resonance frequencies, $f_{n}$, corresponding to the modes $n \lambda$ for a circular loop antenna of diameter $1 \mathrm{~m}$.

\subsection{Transmitting loop antenna}

In this section we run the circular loop antenna in transmitting mode. One of the wire segments are excited with a voltage source with the same shape as the differentiated Gaussian pulse used for the receiving loop antenna. We register the current at this particular wire segment and calculate the input impedance and input admittance according to (21) and (22). We compare with results obtained by the FETD solver developed in [5]. The impedance and admittance are shown in Figure 7. The agreement with the FETD solution is within $1-2 \%$ in the frequency range $60-350 \mathrm{MHz}$, and the consistency of the different loop setups are excellent. The frequency and input impedance at the first full wavelength resonance are collected in Table 3 .
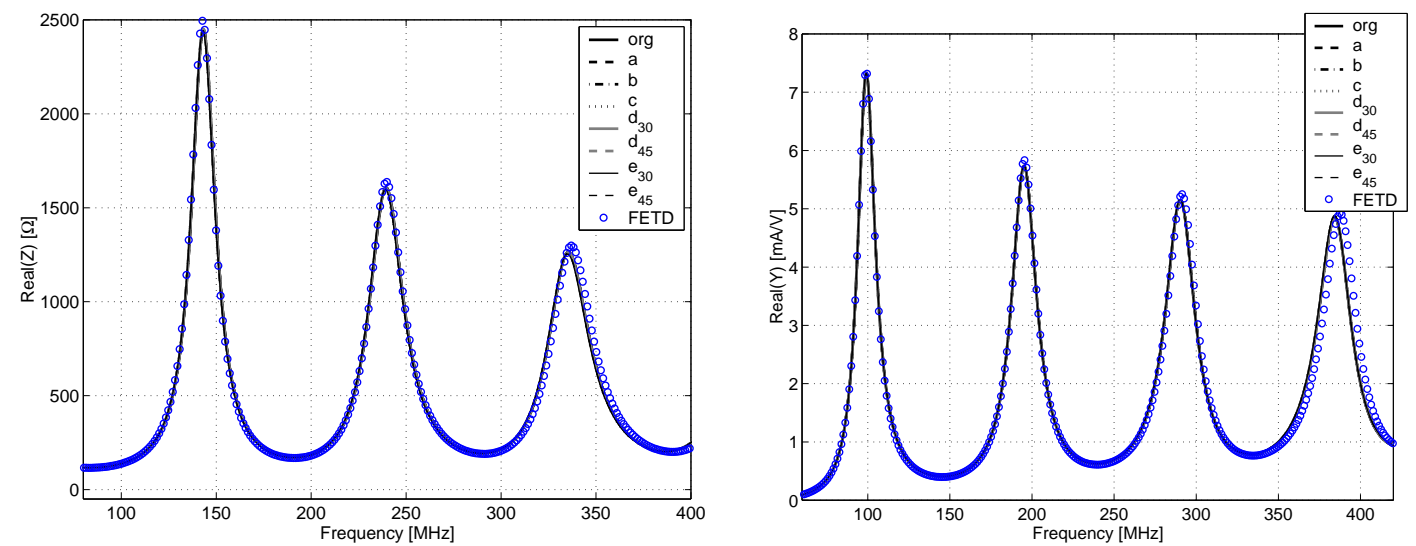

Figure 7: The input resistance (left) and input conductance (right) for the loop.

\subsection{Shielded enclosure}

In [11] measurements were performed on the shielding enclosure geometry shown in Figure 8. The inside of the geometry is discretized with a Cartesian grid with edge lengths $\Delta=1.0 \mathrm{~cm}$. The source is located at the $50 \Omega$ load and has the form

$$
V^{i n c}(t)=e^{-\alpha_{1}^{2}\left(f_{2}-f_{1}\right)^{2}\left(t-\frac{\alpha_{2}}{f_{2}-f_{1}}\right)^{2}} \cos \left[2 \pi \frac{f_{1}+f_{2}}{2}\left(t-\frac{\alpha_{2}}{f_{2}-f_{1}}\right)\right],
$$

where $f_{1}=0.7 \mathrm{GHz}$ and $f_{2}=1.6 \mathrm{GHz}$ are the starting and stopping frequencies of the simulation, respectively, and $\alpha_{1}=1.035$ and $\alpha_{2}=2.539$. The wire has radius $0.08 \mathrm{~cm}$ and is discretized with 14 beams. It is attached to the walls of the geometry at both ends. 


\begin{tabular}{||c||c|c||}
\hline Case & $f[\mathrm{MHz}]$ & real $(\mathrm{Z})[\Omega]$ \\
\hline org & 99.6 & 137.2 \\
$a$ & 99.6 & 137.0 \\
$b$ & 99.6 & 137.2 \\
$c$ & 99.6 & 137.2 \\
$d_{30}$ & 99.5 & 137.1 \\
$d_{45}$ & 99.5 & 137.0 \\
$e_{30}$ & 99.5 & 137.0 \\
$e_{45}$ & 99.5 & 137.0 \\
\hline FETD & 99.4 & 136.3 \\
\hline
\end{tabular}

Table 3: The frequency and resistance at the first full wavelength resonance for a transmitting circular loop of diameter $1 \mathrm{~m}$.

The coax feed line is modeled by the wire through the use of the $50 \Omega$ load. The source and the two loads are placed on the first and last of the wire beams, respectively.

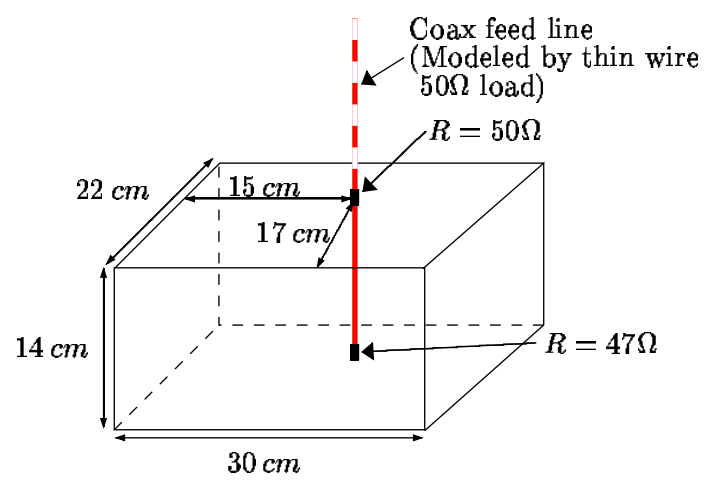

Figure 8: Shielding enclosure geometry.

The time-history of the current at the $50 \Omega$ load, $I_{0}$, and the voltage across the $50 \Omega$ load, $V_{0}$, are stored. A Fast Fourier Transform (FFT) is employed to obtain frequencydomain quantities and roughly 15,000 time steps are needed for a good resolution. The real power delivered to the $50 \Omega$ load is calculated as

$$
\hat{P}=\frac{1}{2} \Re e\left(\frac{\hat{V}_{0}}{\hat{V}^{i n c}} \times \frac{\hat{I}_{0}^{*}}{\hat{V}^{i n c}}\right) .
$$

In Figure 9 we compare the experimental results with our results obtain by the FDTD method. Some deviations are observed around $900 \mathrm{MHz}$, but a very good agreement is observed for the rest of the interval. We note a slight frequency shift of $1 \%$ for the broad feed-probe (TEM) resonance at $1.08 \mathrm{GHz}$. The other resonances show less than $0.5 \%$ deviations between the FDTD and experimental results. Similar frequency shifts have been observed in time-domain integral-equation solutions [12] and FETD solutions [5] for this problem.

\section{Conclusions}

We have presented an extension of the subcell model for thin wires in FDTD proposed by Holland et al. The new interpolation technique makes it possible to accurately model wires not aligned with the edges in the Cartesian grid. The results for different orientations and locations of a dipole antenna show excellent consistency and display good correspondence 


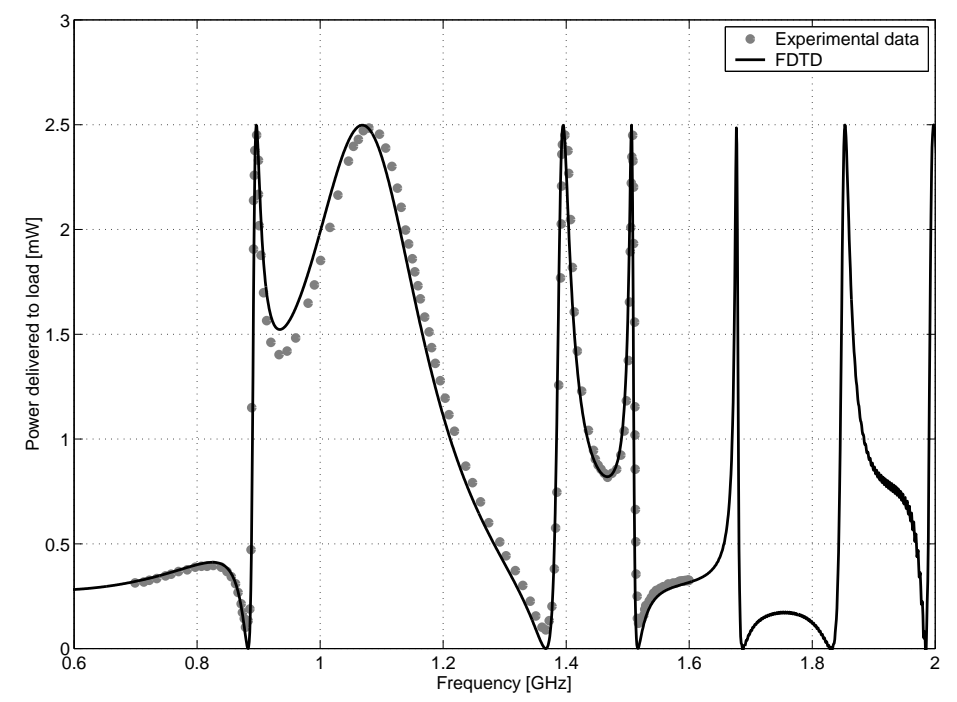

Figure 9: Comparison between FDTD and measurements for a shielding enclosure.

with a Method of Moments solution. Results of good accuracy have also been presented for a loop antenna and a shielding enclosure.

A symmetric coupling between field and wire makes it possible to prove that the resulting time-continuous field-wire system is stable. The fully discrete system is stable under a CFL condition.

A modification of the proposed wire method could be used to generalize the thin-slot model described in [4]. Future work also includes to generalize the proposed method to be able to handle bundled wires.

\section{Acknowledgments}

This work was financed by the Parallel and Scientific Computing Institute (PSCI) and the Swedish Agency for Innovation Systems (VINNOVA).

\section{References}

[1] R. Holland and L. Simpson. Finite-difference analysis of EMP coupling to thin struts and wires. IEEE Trans. Electromagn. Compat., EMC-23(2):88-97, May 1981.

[2] K. R. Umashankar, A. Taflove, and B. Beker. Calculation and experimental validation of induced currents on coupled wires in an arbitrary shaped cavity. IEEE Trans. Antennas Propagat., 35(11):1248-1257, November 1987.

[3] G. Ledfelt. A stable subcell model for arbitrarily oriented thin wires for the FDTD method. International Journal of Numerical Modeling, 15(5), September/October 2002 .

[4] D. J. Riley. Transient finite-elements for computational electromagnetics: Hybridization with finite differences, modeling thin wires and thin slots, and parallel processing. In 17th Annual Review of Progress in Applied Computational Electromagnetics, pages 128-138, Monterey, CA, March 2001.

[5] F. Edelvik, G. Ledfelt, P. Lötstedt, and D. J. Riley. An unconditionally stable subcell model for arbitrarily oriented thin wires in the FETD method. Technical Report 2002-007, Department of Information Technology, Uppsala University, February 2002. 
Available at www.it.uu.se/research/reports, submitted to IEEE Trans. Antennas Propagat.

[6] A. Taflove. Computational Electrodynamics: The Finite-Difference Time-Domain Method. Artech House, Boston, MA, 1995.

[7] J. Jin. The Finite Element Method in Electromagnetics. Wiley, New York, 1993.

[8] B. Gustafsson, H.-O. Kreiss, and J. Oliger. Time Dependent Problems and Difference Methods. Wiley-Interscience, 1995.

[9] F. Edelvik and G. Ledfelt. A comparison of time-domain hybrid solvers for complex scattering problems. International Journal of Numerical Modeling, 15(5), September/October 2002.

[10] C. A. Balanis, editor. Antenna theory: Analysis and Design. John Wiley and Sons, Inc., 2nd edition, 1997.

[11] M. Li, K.-P. Ma, D. M. Hockanson, J. L. Drewniak, T. H. Hubing, and T. P van Doren. Numerical and experimental corroboration of an FDTD thin-slot model for slots near corners of shielding enclosures. IEEE Trans. Electromagn. Compat., 39(3):225-232, August 1997.

[12] E. Michielssen. Personal communication. 